\title{
A Formal Object Definition in Categorical Shaping
}

\author{
Edlira Martiri \\ University of Tirana, Albania \\ E-mail: edlira.martiriunitir.edu.al \\ Edlira Kalemi \\ "Aleksander Moisiu", University of Durres, Albania \\ E-mail: edlirakalemi@uamd.edu.al \\ Gloria Tuxhari \\ University of Tirana, Albania \\ E-mail: gloria.tuxhari@unitir.edu.al
}

Doi:10.5901/ajis.2014.v3n1p427

\section{Abstract}

Formal Representation of Knowledge deals with the construction of real world models taken from a certain domain, which enables automatic reasoning and interpretation. These formal models, called also ontologies, are used to offer formal semantics (forms interpretable by machine) to all kinds of information. Ontology building in Computer Science is tightly connected to its philosophical and logical concepts. Organizing objects into categories is a very important part of knowledge representation. Even though the interaction with the world is made based on individual objects, most of the reasoning is done based on objects' categories. Categorical formation is an intellectual issue even though its objects are not pieces of the intellectual world. As a matter of fact these objects stand at the top of the perceptive world in such a way that they look totally apart from their real structures. Standing at this practical level it is important to cite that the moment of object categorization is a pure formal process. For a business company these categories must reflect business' concepts and rules, its logic and the conventions between the business itself and the organizations it cooperates with. In this paper we build a basis on the formalization of the categorical system. These formalizations are contemporary tools which the Albanian businesses must embrace in order for them to function properly.

Keywords: KBS (Knowledge-Based System), formal representation of knowledge, categorization, logical definition

\section{Introduction to Formal Representation of Knowledge}

Formal theory and predicate logics gives us a potent tool which guarantees reasoning. They also allow us to discuss the borders of our own reasoning and thought, but which can not answer to questions like: "what kind of knowledge should we have to solve a certain problem?", "what is knowledge after all?", "what characteristics does a specific knowledge have" and similar (Jurisica et al, 1999).

Formal Representation of Knowledge is about building real world models of a certain domain or problem, and it enables reasoning and automatic interpretation (Fielding et al., 2004). These formal models, called ontologies, can be used in order to offer formal semantics (i.e. machine interpretable concepts) of every kind of information: database, catalogs, documents and web pages.

A Knowledge Base is a term widely used which has many meanings. We will see the Knowledge Base (KB) as a set of data that has formal semantics. Similarly to ontologies a KB is represented with formalism. It can also include multiple axioms, definitions, rules, facts and other primitives. Differently from ontologies a Knowledge Base' aim is not to represent schemas, base theories or the conceptualization of a domain (Guarino, 2004). In this sense, we could say that ontologies are a special case of knowledge bases.

The organization of objects in categories is a very important part of knowledge representation. Even though the interaction with the world is done via individual objects, the major part of reasoning is done in the categories level. 


\section{Categories and Categorical Shaping}

Of course, the real world is a huge set of objects. In this set, if we see closer, we notice that there is also a huge number of objects' categories. By means of the relational operations we could build structures that are formally defined of a higher order and we can get new objects. The real world materia serves as a base for categorized objects. This process can be extended. All the operations that can be included in the categorical shaping cause objects to behave like subjects and by doing so they shape a category of a higher order. The categorical units can form other relations or operations.

For example an electron belongs to the category "Particle". In this category we have even other objects like protons and neutrons. Particles are part of the category "Matter" e and so on. At the end of our bottom-up categorization the object from which we started is not present any more and it even is not considerable.

The categorical shaping is an entirely intellectual issue, even though the objects with which it deals with are not parts of the intellectual world (Gruber, 2002). These objects stand at the top of the perceptual world so that they can be divided and imparted from all the real structures. According to Smith, the categorical objects are considered "fiat objects" (Guarino, 2004). By this definition, categorical shapes do not relate to form new categories. All the relations, connections, categorizations, etc, just give us new views, since categorical shaping leaves everything as it is (the fact that electrons are divided into categories, that they could have an $\mathrm{X}$ relation to with other categories, this does not effect at all the way how an electron is organized and how it functions in the real world).

Categories play another important role. They organize and simplify the Knowledge Base by means of heritance. First order logics treats the issue of categories by connecting objects with categories or by quantifying compounded elements. We have these statements, as according to (Guarino, 2004):

- An object is an element of a category

- A category is a subclass of another category

- All the elements of a category have properties

- Elements of a category are identified by properties

- The category, as a whole, has its properties.

\section{The Problem of Definition in Ontology}

In general, we refer to an Ontology as a graph which according to Fielding et al. (2004) consists of:

- a set of concepts (graph vertices)

- a set of relations that connect concepts (graph sides)

- a set of instances associated with the concepts

Formally speaking, an Ontology is determined by the function $\mathrm{O}(\mathrm{C}, \mathrm{T}, \mathrm{R}, \mathrm{A}, \mathrm{I}, \mathrm{V})$. It consists of the set of Concepts (C), Types (T), Relations (R), Attributes (A), Instances (I) and Values (V).

Such a theoretical framework can be used to evaluate ontologies as a function that associates a real number to the Ontology (O) (Guarino, 1999).

Building an ontology in computer science, means you first have to understand its concepts which are tightly connected to Philosophy and Logics. First of all you have to understand clearly the domain, then determine unique subjects and their objects and understand and analyze the properties of the objects and their relations. At this practical or engineering level is necessary to highlight that the moment of categorization is a purely formal process.

According to Husserl's theory on categorical shaping every object will be considered part of a certain category. This object will be analyzed and seen part of an upper level category and in this categorization will also be determined its new properties (Russell, 2002). For example, we have the object "plate" and by definition "a plate is a flat dish made of material A, used for B". The object "plate" is part of the category "dish" and has the properties "is flat", "is made of material A" and "is used for B".

Let us continue with our example: "a dish is a container used to carry or transport food". In this case we have a new object, "the dish", which is part of the category "container" and which has some other properties. The object "plate" is considered as the first level and the object "dish" is considered as a second level object. If we continue and go on like this we will define every new category until we will reach the $\mathrm{N}$-th category which does not belong to any category. This category is "The being" or in metaphysics "God", which is defined as "Being is being".

In this case, the given definition and all the other N-levels categories can be treated as a set of axioms, which generate each other. This is not true only in the last case, that of the "Being". For the Being there is no definition, it does not exist an 'axiom'. Then, this is an example that proves the First Theorem of Gödel (Incompleteness Theorem) 
(Penrose, 1999). This theorem states that the system of an Ontology is incomplete, but before let us state this theorem. It expresses two concepts:

(i) The system $S$ is said to be complete formally for the language $G$, if for every sentence $A$ from $G$, either $A$ is provable in $\mathrm{S}$, or not-A is provable in $\mathrm{S}$.

(ii) The system $\mathrm{s}$ is said to be truly complete for the language $\mathrm{G}$, if every true sentence of $\mathrm{G}$ is provable in $\mathrm{S}$.

If $S$ is stable and truly complete then it is formally complete, since every sentence in $G$ is or true or false, so its negation is true.

In (Cocchiarela, 2001) Gödel's theorem, named "Incompleteness Theorem" states that:

"Every logical system that is stable and axiomatic, based on number theory, which states that is complete, will always have statements that can not be proved".

Also, in a formal system, a theorem is not proofed, it is produced. There are two main ways how to operate on this: in the system and for the system. The first way "in the system is a mechanical way and the second one is the intelligent way. If every true statement can be generated by the system, then it is complete. But, every formal system will contain at least one true axiom but which can not be proofed by any mathematical way, nor induction. So, no system is $100 \%$ formalized, not Mathematics, not Logics and even not Ontologies.

\subsection{Algorithmic definition of the "Object Definition"}

To define a term means that this term (object, concept, etc) will be put in a certain category and will be given certain properties. Let us formalize the definition given in (Guarino, 2004).

\subsubsection{Definition:}

We say that the element $X 1$ belongs to the category $X 2$, if it has the properties $A X 21, A X 22, \ldots, A X 2 m$, where $X 21$ to $X 2 m$ are the $m$ - properties of the category $X$ at the second level.

We say that the element $X 2$ belongs to the category $X 3$, if it has the properties $A X 31, A X 32, \ldots, A X 3 m$, where $X 31$ to $X 3 m$ are the $m$ - properties of the category $X$ at the third level.

In this definition we present a new algorithmic way for the formal "object definition".

\subsubsection{Algorithm: Formal object definition}

Begin

Declare $\mathrm{X}_{1}$

For $\mathrm{i}=1$ to $n$

BeginFor

Declare $X_{i+1}$

Belongs $\left(X_{i}, X_{i+1}\right)$

Declare $m$

For $\mathrm{j}=1$ to $\mathrm{m}$

EndFor

HasProperty $\left(X_{i}, A_{x i j}\right)$

EndFor

End

In this algorithm we first assign the object $X$ to the category of the upper level. Than this category is considered as an object itself and this will repeat until we reach the last level of categorization. To every object we assign a set of properties. The procedure HasProperty $\left(\mathrm{X}_{\mathrm{i}}, \mathrm{A}_{\mathrm{ii}}\right)$ does exactly this. It assigns m-properties to the object.

To have a better modeled ontology we must keep in mind that the object analyses might return to a crucial moment for the application. 


\section{Categorical Classification of Sowa}

According to John Sowa (1999), "choosing ontological categories is the first step in the design process of a database, a knowledge base or an object-oriented system". For a bussiness company these categories reflect bussiness concepts and rules, its logic and the conventions between the bussiness and the organizations with which it cooperates with.

Ontological categorization scheme is based on the theories of a large number of philosophers beginning from Plato, Aristotle, Cant, Hegel, Husserl, Whitehead, Heidegger and mostly Pierce. During the whole philosophical route they have treated in different ways the main question: "Which are the categories in which elements of reality take part?" The answer of Sowa to this question come as a result of a very deep analysis and brought to us his main twelve categories, divided in three main groups (Cocchiarela, 2001 and Kephart, 2003):

1. Physical or abstract

It is a division between the physical object which comprises every other thing made of matter or energy and the abstract category for the pure information structures.

2. Continuous or Discrete

The first is an object that has "stable attributes that enable his appearance in different times and places to recognize it as the same object". The second is a process or event which "can be identified from its position in time/space".

3. Firstness, Secondness, Thirdness

An individual can be know as a human being or subtype of it (for example, man or woman) by means of senses (Firstness). The same individual can be classified in base of the relations it creates with other individuals (for example, mother, advocate, employee, etc). This kind of classification depends from the external relations with another entity (Secondness) like kids, clients, work, etc. Thirdness is focused in the medium that enables the relation between the first and the second. Motherhood, including birth and feeding, "connects" a mother to her child, the legal system "creates" the role of the advocate and its relation with the client and the business company "relates" the employees to the managers.

The conceptual analyses and knowledge representation mostly need ontological support development. As a consequence, the activity of ontology development is one of the main steps in knowledge modeling of an organization and is also seen as one of the main pillars of knowledge management.

It is clear that this activity, just as other engineering activities, must be methodologically coherent in its phases. Even though the development of software applications which offer automatization in such modeling, the results from tools for automatic ontologies in the real practice of knowledge management are a disappointing.

\section{Conclusions and Recommendations}

In this paper we presented an overview of the concept of object categorization and its importance in information theory. In this case categorical formation is an intellectual issue, even though its objects are not pieces of the intellectual world. We conducted a structured way and came with a formal definition of the "object definition" itself, since they are the main building pieces of our perceptual world. The algorithmic form that we introduced is machine-interpretable and helps in information processing of objects.

We recommend to implement this algorithm in ontology building and also to extend even more these logical implications of this theory.

\section{References}

Cocchiarella, Nino B., (2001), Logic And Ontology, Kluwer Academic Publishers, Netherlands.

Fielding, J., Simon J., Ceusters W., Smith B. (2004). Ontological theory for ontological engineering. Proceedings of the Ninth International Conference on the Principles of Knowledge Representation and Reasoning. Whistler.

Gruber, Thomas R., (2002) Toward Principles for the Design of Ontologies Used for Knowledge Sharing - Some issues concerning the ontology development process, IEEE Computer Society.

Guarino, N., (2001). Ontologies and Knowledge Bases - Towards a Terminological Clarification National Research Council, LADSEBCNR.

Guarino, N., (2004). "Formal Ontology, Conceptual Analysis and Knowledge Representation", International Conference, Florence.

Jurisica I., Mylopoulos J., Yu E. (1999), Using Ontologies for Knowledge Management: An Information Systems Perspective - Annual Conference of the American Society for Information Science, Washington, D.C.. 
Kephart, Jeffrey O.; Chess, David M., (2003). The Vision of Autonomic Computing - published by the IEEE Computer society. Penrose, R.: "Shadows of the mind", 1999.

Russell, B., (2002). Artificial Intelligence: a modern approach, Wiley.

Zhylia, D., (1994). Larousse - Fjalor i filozofise, Shtepia Botuese Enciklopedike, Tirane, Albania 
